\title{
Correction to: Critical Evaluation and Optimization of the Fe-N, Mn-N and Fe-Mn-N Systems
}

\author{
Zhimin You $^{1} \cdot$ Min-Kyu Paek ${ }^{2}$ In-Ho Jung ${ }^{2}$
}

Published online: 26 October 2018

(C) ASM International 2018

Correction to: J. Phase Equilib. Diffus. (2018) 39:650-677

https://doi.org/10.1007/s11669-018-0666-8

In the originally published article, numbers in Eq 2 and 3 were transposed. The following are the full, correct equations:

$$
\begin{aligned}
& H_{T}^{o}=\Delta H_{298.15 K}^{o}+\int_{T=298.15 K}^{T} C_{P} \mathrm{~d} T \\
& S_{T}^{o}=S_{298.15 K}^{o}+\int_{T=298.15 K}^{T}\left(C_{P} / T\right) \mathrm{d} T
\end{aligned}
$$

The original article can be found online at https:// doi.org/10.1007/s11669-018-0666-8.

In-Ho Jung

in-ho.jung@snu.ac.kr

1 Department of Mining and Materials Engineering, McGill University, 3610 University Street, Montreal, QC H3A 0C5, Canada

2 Department of Materials Science and Engineering, Research Institute of Advanced Materials (RIAM), Seoul National University, 1 Gwanak-ro, Gwanak-gu, Seoul 08826, South Korea 